\title{
Relação entre morbilidade psicológica, variáveis sociodemográficas e clínicas, perceção de intimidade relacional, satisfação conjugal e preocupações sentidas durante a gravidez
}

Eva Castanheira, ${ }^{1}$ Paulo Correia, ${ }^{2}$ Eleonora C. V. Costa ${ }^{3}$

\section{RESUMO}

Objetivos: Avaliar a relação entre variáveis sociodemográficas e clínicas, perceção de intimidade relacional, satisfação conjugal, preocupações sentidas durante a gravidez e morbilidade psicológica (ansiedade, depressão e stress).

Tipo de estudo: Observacional, transversal.

Local: ACeS da zona norte do País (Barcelos/Esposende - ACeS Cávado III).

População: Grávidas no segundo e terceiro trimestres de gravidez.

Métodos: A amostra foi recolhida nas sessões de preparação para o parto efetuadas no ACeS Cávado III, tendo as utentes respondido a um questionário.

Resultados: Maiores preocupações associam-se a maior morbilidade psicológica, menor perceção de intimidade relacional e satisfação conjugal associam-se a maior morbilidade psicológica. A perceção de intimidade relacional relacionou-se positivamente com a satisfação conjugal e negativamente com as preocupações.

Conclusões: A gravidez pode comportar consideráveis riscos para a saúde mental da mulher.

Palavras-chave: Gravidez; Ansiedade; Depressão; Stress.

\section{INTRODUÇÃO}

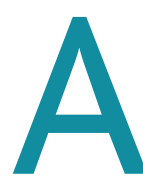

gravidez e transição para a parentalidade é, geralmente, uma condição desejada e programada pelo casal, sendo concebida e imaginada como um processo positivo e saudável para ambos e inclusive potenciador da satisfação conjugal. ${ }^{1}$ Porém, e enquanto experiência emocional complexa, a

1. Departamento de Psicologia, Universidade Católica Portuguesa 2. ACeS do Cávado III - Centro de Saúde de Esposende.

3. Departamento de Psicologia, Universidade Católica Portuguesa, ACeS do Cávado III - Centro de Saúde de Barcelos/Barcelinhos. gravidezé também caracterizada como uma das mais difíceis e stressantes transições da vida do ser humano. De facto, o nascimento de um filho pode estar associado tanto a emoções positivas como negativas para a mulher, levando a que, aquando da gravidez, cerca de $10 \%$ das mulheres a nível mundial padeça de doenças mentais, especialmente estados depressivos e ansiosos. Esta proporção é relativa a mulheres grávidas e é inferior à das mulheres não grávidas, controlando fatores sociodemográficos $^{2-3}$ e sendo o suicídio uma das principais causas de morte relacionadas com a gravidez. ${ }^{2}$ Apesar das evi- 
dências observadas na literatura, o período da gravidez pode também associar-se a uma melhoria da saúde mental da mulher, que pressupõe desenvolvimento e crescimento, nomeadamente ao nível pessoal e social. ${ }^{4}$ Todavia, esta é vivenciada como um período crítico, que contribui para o desenvolvimento do stress e de perturbações ansiosas e depressivas, comprometendo consequentemente a saúde mental da mulher grávida. ${ }^{5-12}$

O período da gravidez é ainda associado a uma etapa de vida em que as mulheres experienciam necessidades superiores de apoio, sendo o parceiro íntimo o principal prestador de auxílio neste período, percecionado como capaz de proporcionar o apoio necessário numa situação de adaptação. De facto, as grávidas esperam que os seus parceiros sejam capazes de providenciar suporte emocional, físico e económico aquando da gravidez, ${ }^{13}$ sendo que este suporte assume características protetoras da saúde mental da mulher, reduzindo os níveis de stress e encorajando comportamentos maternos mais positivos. ${ }^{14-16}$ Neste sentido, grávidas com maior perceção de suporte do parceiro experienciam níveis mais reduzidos de distresse emocional durante e após a gravidez, assim como os seus outros filhos irão manifestar níveis mais elevados de tolerância à novidade aquando do nascimento e nos primeiros meses de vida. ${ }^{15}$

Sendo o suporte do parceiro um fator protetor para a saúde mental da mulher grávida, também a satisfação conjugal se assume como uma variável protetora. De facto, a satisfação conjugal, anterior à gravidez, constitui-se como um forte determinante para a saúde mental da grávida, ${ }^{1,8,17-18}$ uma vez que quando existe uma insatisfação conjugal prévia o casal experiencia, durante a gravidez, um agravamento da insatisfação conjugal e o desenvolvimento de sintomas psicopatológicos, como a ansiedade e a depressão. ${ }^{6,8,10,12}$ No entanto, e apesar de a gravidez produzir alterações na qualidade da satisfação conjugal, nomeadamente o declínio da qualidade relacional e a insatisfação com o relacionamento, estas alterações podem ser experienciadas com diferentes intensidades dependendo de cada casal. ${ }^{1,17} \mathrm{~A}$ satisfação conjugal prévia à gravidez, ${ }^{1,8,10,17-18}$ o grau em que a gravidez foi planeada ${ }^{17-18} \mathrm{e}$ o estilo de vinculação que a mulher ${ }^{19} \mathrm{e}$ o seu companheiro ${ }^{20}$ têm, antes e após a gravidez, são considerados fatores protetores da satisfação conjugal.
A gravidez enquanto período de transição de vida revela-se, como referenciado anteriormente, exigente na medida em que a mulher se preocupa com maior intensidade e frequência, sendo geralmente as preocupações sentidas durante a gravidez de caráter normativo, cujo conteúdo versa questões médicas, relacionais, de perda reprodutiva, da saúde, sociais e económicas. ${ }^{21-26} \mathrm{Na}$ literatura encontram-se diversos estudos que procuraram caracterizar as preocupações sentidas durante a gravidez em relação ao conteúdo e intensidade, evidenciando-se maioritariamente variáveis como a idade, ${ }^{24,27}$ diferenças étnicas, ${ }^{28}$ número de gravidezes anteriores, ${ }^{23,26,29}$ níveis de ensino, rendimento familiar, maturidade emocional da mulher grávida ${ }^{24} \mathrm{e}$ suas características individuais (e.g., estratégias de $c o$ ping; características da personalidade), ${ }^{30-31}$ a saúde do bebé, o tipo de parto, ${ }^{26}$ problemas financeiros, possibilidade de aborto $^{22} \mathrm{e}$ o medo de inadequação às tarefas maternas e inadequação do suporte social ${ }^{32}$ como preocupações comummente associadas ao período gravídico e as quais potenciam o desenvolvimento de sintomatologia psicológica. Com base num estudo realizado em Portugal constata-se que as preocupações na gravidez seguem a seguinte ordem de relevância: questões médicas, socioeconómicas, profissionais e familiares, designadamente possibilidade de ocorrência de complicações na gravidez, aborto espontâneo, diagnóstico de malformações, existência de problemas financeiros, baixo rendimento económico, possibilidade de perda de emprego, morte de um familiar e ocorrência de problemas graves na família. ${ }^{21}$

Diversos estudos têm verificado que variáveis como o estatuto marital, o planeamento da gravidez, a sobrecarga de funções ${ }^{33} \mathrm{e} o$ estilo de vinculação ${ }^{19}$ têm forte influência na predição da satisfação parental, levando a uma melhor regulação das emoções negativas associadas a este período. Por sua vez, e em consonância com estes dados, os fatores relacionais, ${ }^{34}$ fatores sociais (e.g., residência rural, pobreza, exposição à violência familiar), ${ }^{35-37}$ a paridade ${ }^{11,38-42} \mathrm{e}$ fatores sociodemográficos (e.g., idade ${ }^{43}$ constituem-se como fortes fatores determinantes para o desenvolvimento de sintomas psicopatológicos na mulher grávida.

Observa-se na literatura uma ampla variedade de estudos que procuraram compreender o efeito da paridade nas variáveis psicológicas, sociocognitivas e clí- 
nicas aquando da gravidez, verificando-se que as mulheres primíparas percecionam maior intimidade relacional aquando da gravidez do que as mulheres que já vivenciaram outra gravidez. ${ }^{41-42}$

A literatura indica o potencial benefício das intervenções psicológicas que se focam na diminuição das preocupações sentidas durante a gravidez e na promoção de comportamentos (e.g., competências de comunicação) que aumentam o nível de intimidade nos casais como forma de melhorar o bem-estar psicológico na mulher grávida. ${ }^{24,60}$

Em suma, considera-se que a perceção de intimidade relacional, a satisfação conjugal e as preocupações sentidas durante a gravidez assumem um forte impacto na saúde mental da mulher aquando da gravidez, podendo essa relação ser minimizada e/ou maximizada pela ação das variáveis sociodemográficas e clínicas. Assim, a presente investigação pretende avaliar a relação entre variáveis sociodemográficas e clínicas, perceção de intimidade relacional, satisfação conjugal, preocupações sentidas durante a gravidez e morbilidade psicológica (ansiedade, depressão e stress).

\section{MÉTODOS}

O estudo adota a modalidade de investigação científica quantitativa, designadamente um desenho do tipo observacional analítico-transversal.

A amostra $(n=200)$ constitui uma amostra de conveniência, não probabilística, foi recolhida nas sessões de preparação para o parto realizadas no ACeS Cávado III - Barcelos/Esposende entre os meses de junho e dezembro de 2014. As participantes elegíveis para integrar o estudo foram identificadas pela equipa de enfermagem responsável pelas sessões de preparação para o parto.

Os critérios de participação foram: estar grávida, frequentar as sessões de preparação para o parto e possuir competências básicas de escrita e leitura. Os critérios de exclusão consistiram na ausência de patologia e psicopatologia e na inexistência de gravidez de risco.

No total, 200 grávidas (média=31 semanas de gestação) aceitaram colaborar na investigação. Assim, após as sessões de preparação para o parto, as grávidas foram encaminhadas para uma sala privada onde foram recebidas pelos investigadores que lhes explicavam o caráter voluntário e confidencial da participação, forneciam in- formação adicional sobre as tarefas a realizar e esclareciam as dúvidas existentes. Posteriormente as grávidas preenchiam o consentimento informado e o questionário de estudo. A recolha tinha a duração aproximada de 30 minutos e as participantes não foram pagas.

Para a recolha dos dados sociodemográficos, obstétricos e clínicos foi concebido e utilizado um questionário, adaptado à população em estudo, que incluiu: idade, estado civil, duração do estado civil, habilitações literárias, rendimento pessoal anual, estatuto profissional, tempo de gestação, paridade, planeamento da gravidez, complicações durante a gravidez e história de aborto.

O Personal Assessment of Intimacy in Relationships Scale (PAIR) ${ }^{44}$ é um questionário de 36 itens concebido para avaliar a intimidade relacional numa escala lickert de cinco pontos $(0=$ Discordo fortemente a $5=$ Concordo fortemente). Resultados mais elevados indicam níveis mais altos de intimidade relacional. Os totais variam entre 0 e 144 . O questionário foi adaptado para a população portuguesa, sendo encontradas boas características psicométricas. ${ }^{45}$ Neste estudo, a consistência interna na escala total do instrumento foi de .91.

A Escala de Avaliação da Satisfação em Áreas da Vida Conjugal (EASAVIC ${ }^{46}$ é um questionário de autopreenchimento, composto por 44 itens, que pretende avaliar a perceção subjetiva que cada indivíduo tem da sua relação, casamento ou união de facto, numa escala lickert de seis pontos ( $1=$ Nada satisfeito/a e 6=Completamente satisfeito/a). Os valores totais variam entre 1 e 6; resultados elevados indicam maior perceção de satisfação conjugal. O questionário foi concebido para a população portuguesa, sendo encontradas boas características psicométricas. ${ }^{46}$ Nesta amostra, a consistência interna foi de 0,97 .

A Cambridge Worry Scale (CWS) ${ }^{23}$ tem 16 itens que permitem avaliar o conteúdo e a intensidade das preocupações, conceptualizadas como normativas, sentidas durante a gravidez, numa escala lickert de seis pontos entre 0 («Não é uma preocupação») e 5 («É uma grande preocupação»). Os totais variam entre 0 e 80; valores elevados indicam maior número e/ou intensidade de preocupações. O questionário foi adaptado para a população portuguesa, sendo encontradas boas características psicométricas ${ }^{47}$ Neste estudo, a consistência interna na escala total do instrumento foi de 0,86 . 
As Depression, Anxiety, and Stress Scale - 21 items $\left(\right.$ DASS-21) ${ }^{48}$ são um conjunto de três escalas de autopreenchimento, com 21 itens divididos em igual número pelas três escalas, desenvolvidas para avaliar os estados emocionais de depressão, ansiedade e stress numa escala lickert de quatro pontos ( $0=$ Não se aplicou nada a mim a $4=$ Aplicou-se a mim a maior parte das vezes). Os valores totais das subescalas variam entre 0 e 28 e são alcançados através da soma dos resultados dos itens, sendo que totais mais elevados correspondem a estados afetivos mais negativos. A versão portuguesa da DASS-21 apresentou bons resultados ao nível das características psicométricas. ${ }^{49}$ No presente estudo, o coeficiente de consistência interna foi adequado para a Depressão $(\alpha=0,86)$, para a Ansiedade $(\alpha=0,83)$ e para o stress $(\alpha=0,88)$.

As análises estatísticas foram executadas utilizando o software IBM SPSS v. 22. Foram realizadas análises descritivas das variáveis demográficas, clínicas, obstétricas e psicológicas, sendo calculados a média e o desvio-padrão para as variáveis contínuas e descritas as frequências absolutas e relativas para as variáveis categóricas. Adicionalmente foram realizados testes $t$ (para variáveis contínuas) e testes de qui-quadrado (para variáveis nominais) para comparar as variáveis demográficas obstétricas e psicológicas entre grávidas multíparas e primíparas. Para verificar as correlações entre a morbilidade psicológica (depressão, ansiedade e stress) e as variáveis psicológicas e relacionais foi usado o teste Coeficiente de Correlação de Spearman's Rho.

\section{RESULTADOS}

As características clínicas e demográficas das participantes podem ser observadas no Quadro I. A idade das participantes variou entre os 17 e os 41 anos (média $=30,2 ; \mathrm{DP}=4,4), 86 \%$ da amostra é casada ou vive em união de facto (a duração da relação variou entre um e 14 anos; média=4,2, DP=2,6) e 14\% das grávidas são solteiras. Oitenta e nove e meio por cento das mulheres possuem uma relação marital e $10,5 \%$ não tem relação marital. Trinta e seis por cento da amostra possui um grau de ensino superior, $37 \%$ o ensino médio ou secundário e $28 \%$ possui o ensino básico/elementar. Aquando da recolha de dados, $12,8 \%$ encontrava-se desempregada e $44,1 \%$ encontrava-se em situação de incapacidade temporária. As restantes $(43,1 \%)$ encontra- vam-se profissionalmente ativas, $46,5 \%$ auferia um rendimento anual inferior $\mathrm{a} € 6.000,41 \%$ entre $€ 6.000 \mathrm{e} €$ 12.000 e $12,5 \%$ auferia rendimentos superiores $\mathrm{a} €$ 12.000. Setenta e sete e meio por cento das participantes encontrava-se grávida pela primeira vez (o tempo de gestação variava entre as 12 e 39 semanas; média $=31,4, \mathrm{DP}=5$ ), $22,5 \%$ era multípara e $16,5 \%$ das mulheres experienciaram complicações durante a gravidez, nomeadamente tensão arterial $(15,2 \%)$, diabetes $(27,3 \%)$, hemorragias $(18,2 \%)$ e outos problemas não especificados (39\%).

Ao nível das variáveis psicológicas observou-se que o valor médio para o PAIR foi de 90,89 ( $\mathrm{DP}=13,69)$, variando entre 45 e 119; para a EASAVIC foi de 4,59 $(\mathrm{DP}=0,68)$, variando entre três e seis; para a CWS foi de $1,56(\mathrm{DP}=0,88)$, variando entre zero e 4,31 ; para a Ansiedade (subescala da DASS-21) foi de 5,69 ( $\mathrm{DP}=6,80$ ), variando entre zero e 38; para a Depressão (subescala da DASS-21) foi de 3,99 ( $\mathrm{DP}=5,60)$, variando entre zero e 32; por último, para o Stress (subescala da DASS-21) foi de $10,74(\mathrm{DP}=8,17)$, variando entre zero e 38 .

Foram observadas diferenças estatisticamente significativas entre grávidas primíparas e multíparas ao nível da idade, duração do estado civil, habilitações, rendimento anual, tempo de gestação, satisfação conjugal, preocupações sentidas durante a gravidez e dos sintomas de depressão, ansiedade e stress (Quadro I).

O Quadro II apresenta as correlações existentes entre os sintomas de ansiedade, depressão e stress, as preocupações sentidas durante a gravidez, a satisfação conjugal e a perceção de intimidade relacional.

Através das análises realizadas (conferir Quadro II) verificou-se que existe uma relação positiva significativa entre a morbilidade psicológica e as preocupações sentidas durante a gravidez, nomeadamente na ansiedade $(r=0,298, p<0,01)$, na depressão $(r=0,255, p<0,01)$ e no stress $(r=0,344, p<0,01)$, evidenciando que a uma maior morbilidade psicológica está associado um maior número e/ou intensidade de preocupações sentidas durante a gravidez.

Verificaram-se também relações negativas significativas entre a morbilidade psicológica e a perceção de intimidade relacional, designadamente na ansiedade $(r=-0,239, p<0,01)$, na depressão $(r=-0,324, p<0,01)$ e no stress $(r=-0,179, p<0,05)$, evidenciando que maior morbilidade psicológica está associada a menor perceção 


\begin{tabular}{|c|c|c|c|c|c|c|c|}
\hline & \multicolumn{2}{|c|}{$\begin{array}{l}\text { Coorte } \\
n=200\end{array}$} & \multicolumn{2}{|c|}{$\begin{array}{c}\text { Primíparas } \\
n=155\end{array}$} & \multicolumn{2}{|c|}{$\begin{array}{c}\text { Multíparas } \\
n=45\end{array}$} & \multirow[t]{2}{*}{$P^{\mathrm{a}}$} \\
\hline & M & DP & M & DP & M & DP & \\
\hline Variáveis sociodemográficas & $n$ & $\%$ & $n$ & $\%$ & $n$ & $\%$ & \\
\hline Idade & 30.24 & 4.41 & 29.75 & 4.25 & 31.93 & 4.59 & .020 \\
\hline Duração da relação conjugal & 4.22 & 2.64 & 3.81 & 2.30 & 5.71 & 3.24 & .000 \\
\hline $\begin{array}{l}\text { Estado civil } \\
\text { Casada } \\
\text { União de facto } \\
\text { Solteira }\end{array}$ & $\begin{array}{r}136 \\
36 \\
28\end{array}$ & $\begin{array}{l}68.0 \\
18.0 \\
14.0\end{array}$ & $\begin{array}{l}95 \\
33 \\
27\end{array}$ & $\begin{array}{l}61.3 \\
21.3 \\
17.4\end{array}$ & $\begin{array}{r}41 \\
3 \\
1\end{array}$ & $\begin{array}{r}91.1 \\
6.7 \\
2.2\end{array}$ & .001 \\
\hline $\begin{array}{l}\text { Habilitações literárias } \\
\text { Até ao } 9^{\circ} \text { ano } \\
12^{\circ} \text { ano } \\
\text { Ensino superior }\end{array}$ & $\begin{array}{l}55 \\
73 \\
71\end{array}$ & $\begin{array}{l}27.6 \\
36.7 \\
35.7\end{array}$ & $\begin{array}{l}36 \\
59 \\
60\end{array}$ & $\begin{array}{l}23.2 \\
38.1 \\
38.7\end{array}$ & $\begin{array}{l}19 \\
14 \\
11\end{array}$ & $\begin{array}{r}43.2 \\
31.8 \\
25\end{array}$ & .029 \\
\hline $\begin{array}{l}\text { Rendimento anual } \\
\text { Inferior } a € 6.000 \\
\text { Entre } € 6.000-12.000 \\
\text { Superior } a € 12.000\end{array}$ & $\begin{array}{l}93 \\
82 \\
25\end{array}$ & $\begin{array}{l}46.5 \\
41.0 \\
12.5\end{array}$ & $\begin{array}{l}79 \\
56 \\
20\end{array}$ & $\begin{array}{r}51 \\
36.1 \\
12.9\end{array}$ & $\begin{array}{r}14 \\
26 \\
5\end{array}$ & $\begin{array}{l}31.1 \\
57.8 \\
11.1\end{array}$ & .030 \\
\hline $\begin{array}{l}\text { Situação profissional } \\
\text { Empregada } \\
\text { Desempregada } \\
\text { Baixa médica } \\
\end{array}$ & $\begin{array}{l}84 \\
25 \\
86\end{array}$ & $\begin{array}{l}43.1 \\
12.8 \\
44.1\end{array}$ & $\begin{array}{l}67 \\
20 \\
64\end{array}$ & $\begin{array}{l}44.4 \\
13.2 \\
42.4\end{array}$ & $\begin{array}{r}17 \\
5 \\
22 \\
\end{array}$ & $\begin{array}{l}37.8 \\
11.1 \\
48.9\end{array}$ & .670 \\
\hline $\begin{array}{l}\text { Variáveis clínicas } \\
\text { Tempo de gravidez (semanas) }\end{array}$ & 31.4 & 5.0 & 31.48 & 4.88 & 31.20 & 5.497 & .015 \\
\hline $\begin{array}{l}\text { Planeamento da gravidez } \\
\text { Apenas pela mãe } \\
\text { Por ambos } \\
\text { Não planeada }\end{array}$ & $\begin{array}{r}1 \\
179 \\
20\end{array}$ & $\begin{array}{r}0.5 \\
89.5 \\
10\end{array}$ & $\begin{array}{r}1 \\
138 \\
16\end{array}$ & $\begin{array}{r}0.6 \\
89 \\
10.3\end{array}$ & $\begin{array}{r}0 \\
41 \\
4\end{array}$ & $\begin{array}{r}0 \\
91.1 \\
8.9\end{array}$ & .827 \\
\hline $\begin{array}{l}\text { Complicações durante a gravidez } \\
\text { Sim } \\
\text { Não }\end{array}$ & $\begin{array}{r}33 \\
167\end{array}$ & $\begin{array}{l}16.5 \\
83.5\end{array}$ & $\begin{array}{r}26 \\
129\end{array}$ & $\begin{array}{l}16.8 \\
83.2\end{array}$ & $\begin{array}{r}7 \\
38\end{array}$ & $\begin{array}{l}15.6 \\
84.4\end{array}$ & .525 \\
\hline $\begin{array}{l}\text { Problemas de saúde durante a gravidez } \\
\text { Hipertensão } \\
\text { Diabetes } \\
\text { Hemorragias } \\
\text { Outros problemas de saúde }\end{array}$ & $\begin{array}{r}5 \\
9 \\
6 \\
13\end{array}$ & $\begin{array}{l}15.2 \\
27.3 \\
18.2 \\
39.4\end{array}$ & $\begin{array}{l}5 \\
7 \\
4 \\
9\end{array}$ & $\begin{array}{l}20 \\
28 \\
16 \\
36\end{array}$ & $\begin{array}{l}0 \\
2 \\
2 \\
4\end{array}$ & $\begin{array}{r}0 \\
25 \\
25 \\
50\end{array}$ & .534 \\
\hline $\begin{array}{l}\text { História de aborto } \\
\text { Sim } \\
\text { Não }\end{array}$ & $\begin{array}{r}22 \\
178\end{array}$ & $\begin{array}{l}11 \\
89\end{array}$ & $\begin{array}{r}0 \\
155\end{array}$ & $\begin{array}{r}0 \\
100\end{array}$ & $\begin{array}{l}22 \\
23\end{array}$ & $\begin{array}{l}48.9 \\
51.1\end{array}$ & .000 \\
\hline
\end{tabular}

a Estimado por testes $t$ para diferenças de média e testes de Pearson para a independência.

Notas: M - Média; DP - Desvio-Padrão. 


\begin{tabular}{|c|c|c|c|c|c|c|c|}
\hline & \multicolumn{2}{|c|}{$\begin{array}{l}\text { Coorte } \\
n=200\end{array}$} & \multicolumn{2}{|c|}{$\begin{array}{c}\text { Primíparas } \\
n=155\end{array}$} & \multicolumn{2}{|c|}{$\begin{array}{c}\text { Multíparas } \\
n=45\end{array}$} & \multirow[t]{2}{*}{$P^{\mathrm{a}}$} \\
\hline & M & DP & M & DP & $M$ & DP & \\
\hline Variáveis sociodemográficas & $n$ & $\%$ & $n$ & $\%$ & $n$ & $\%$ & \\
\hline \multicolumn{8}{|l|}{ Variáveis psicológicas } \\
\hline Perceção de intimidade relacional & 90.89 & 13.69 & 90.61 & 13.96 & 91.86 & 12.85 & .594 \\
\hline Satisfação conjugal & 4.69 & 0.68 & 4.69 & 0.64 & 4.70 & 0.79 & .000 \\
\hline Preocupações durante a gravidez & 1.56 & 0.88 & 1.59 & 0.89 & 1.46 & 0.85 & .003 \\
\hline Depressão & 3.99 & 5.60 & 3.86 & 5.71 & 4.44 & 5.26 & .008 \\
\hline Ansiedade & 5.69 & 6.80 & 5.21 & 6.62 & 7.33 & 7.24 & .018 \\
\hline Stress & 10.74 & 8.17 & 10.6 & 7.81 & 11.20 & 9.39 & .000 \\
\hline
\end{tabular}

\begin{tabular}{|c|c|c|c|c|c|c|}
\hline & $\begin{array}{c}\text { Perceção de } \\
\text { intimidade } \\
\text { relacional }\end{array}$ & $\begin{array}{l}\text { Satisfação } \\
\text { conjugal }\end{array}$ & $\begin{array}{c}\text { Preocupações } \\
\text { sentidas } \\
\text { durante a } \\
\text { gravidez }\end{array}$ & Ansiedade & Depressão & Stress \\
\hline \multicolumn{7}{|l|}{$\begin{array}{l}\text { Perceção de intimidade } \\
\text { relacional }\end{array}$} \\
\hline Satisfação conjugal & $.711^{* *}$ & 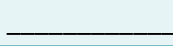 & & & & \\
\hline $\begin{array}{l}\text { Preocupações sentidas } \\
\text { durante a gravidez }\end{array}$ & $-.207^{* *}$ & $-.237^{* *}$ & & & & \\
\hline Ansiedade & $-.239 * *$ & $-.175^{*}$ & $.298^{* *}$ & & & \\
\hline Depressão & $-.324^{* *}$ & $-.214^{* *}$ & $.255^{* *}$ & $.626^{* *}$ & & \\
\hline Stress & $-.179 *$ & $-.165^{*}$ & $.344^{* *}$ & $.698 * *$ & $.634^{* *}$ & \\
\hline
\end{tabular}

${ }^{* *} p<.01 ;{ }^{*} p<.05$

de intimidade relacional por parte da grávida. Foram ainda observadas relações negativas significativas face à satisfação conjugal, na ansiedade $(r=-0,175, p<0,05)$, na depressão $(r=-0,214, p<0,01)$ e no stress $(r=-0,165$, $p<0,05)$, sendo que a morbilidade psicológica está associada à insatisfação conjugal.

\section{DISCUSSÃO}

Na presente amostra não foram verificadas diferenças ao nível da perceção de intimidade relacional entre as participantes, observando-se valores médios similares entre primíparas e multíparas, contrariamente ao verificado noutros estudos. ${ }^{41-42}$
Através da realização de uma meta-análise, alguns autores concluíram a existência de efeitos na satisfação conjugal face à primeira gravidez, observando um impacto negativo na satisfação conjugal.$^{18}$ Outros estudos observaram que, aquando da gravidez, as mulheres multíparas experienciam maior insatisfação conjugal que as primíparas. ${ }^{50} \mathrm{De}$ facto, os dados alcançados com o presente estudo revelam-se discordantes dos observados na literatura, na medida em que não foram observadas diferenças estatisticamente significativas entre as participantes ao nível dos efeitos da paridade na satisfação conjugal.

Relativamente às preocupações sentidas durante a 
gravidez, a literatura identifica a existência de diferenças estatisticamente significativas ao nível da paridade, sendo que mulheres primíparas tendem a evidenciar maiores preocupações que as multíparas..$^{23,26,29}$ Contrariamente ao exposto, não foram observadas diferenças no presente estudo.

Ao nível da morbilidade psicológica, os dados encontrados na literatura não são unânimes, na medida em que se observa a existência de estudos que revelaram que a paridade assume diferenças ao nível da morbilidade psicológica da mulher grávida ${ }^{11,38-40,51}$ e estudos que observaram a inexistência de diferenças ao nível da morbilidade psicológica entre mulheres multíparas e primíparas. ${ }^{12,52-57} \mathrm{O}$ presente estudo demonstra-se parcialmente concordante com a literatura, uma vez que foram observadas diferenças entre as participantes primíparas e multíparas apenas ao nível da sintomatologia ansiosa, à semelhança do que se verificou noutras investigações, ${ }^{11,15,38,51}$ não se observando diferenças entre as participantes no que concerne à depressão e ao stress, de modo também consonante com a literatura. ${ }^{12,51-54} \mathrm{O}$ aumento da ansiedade em mulheres com outros filhos pode estar associado a preocupações relativas à aceitação e às responsabilidades maternas face aos filhos pré-existentes. De facto, apesar do conhecimento prévio associado ao processo de gravidez, as mulheres multíparas experienciam outras preocupações, designadamente a necessidade de adequar as tarefas maternas, o aumento das responsabilidades e preocupações e o aumento das despesas associadas aos filhos.

De facto, a gravidez envolve grandes mudanças biológicas e psicossociais na vida da mulher, constituindo um momento em que as preocupações assumem maior ênfase e intensidade. De facto, o conteúdo das preocupações assume um caráter normativo, diferenciando-se assim do constructo de ansiedade..$^{23}$ Observou-se, porém, na literatura que as preocupações assumem consideráveis repercussões ao nível da morbilidade psicológica. ${ }^{22,58}$ Observa-se que as preocupações que as grávidas possuem durante a gravidez assumem consideráveis implicações no desenvolvimento de sintomatologia depressiva ${ }^{59} \mathrm{e}$ ansiosa. ${ }^{30} \mathrm{O}$ presente estudo demonstrou-se consonante com a literatura, dado que foi observada uma relação positiva significativa e recíproca entre as preocupações sentidas durante a gravidez e a morbilidade psicológica, como foi observado noutras investigações. ${ }^{30,59}$

De outro modo, se as preocupações sentidas durante a gravidez predispõem a mulher ao desenvolvimento de morbilidade psicológica, a perceção de intimidade relacional bem como a satisfação conjugal revelamse como variáveis protetoras da mulher neste período. De facto, os dados observados na literatura indicam que a perceção de intimidade relacional, aquando da gravidez, assume características protetoras da saúde mental da mulher, reduzindo os níveis de morbilidade psicológica. ${ }^{13-16}$ Estes dados são consonantes com os alcançados na presente amostra, em que níveis mais elevados de perceção de intimidade relacional correspondem a menor morbilidade psicológica.

Observou-se também, no presente estudo, a existência de uma relação negativa entre a satisfação conjugal e a morbilidade psicológica aquando da gravidez, sendo estes dados concordantes com outros estudos. ${ }^{6,8,10,12,34,50}$ Particularmente neste estudo, a satisfação conjugal associa-se à existência de saúde mental. De facto, considera-se que a vivência saudável dos relacionamentos, designadamente a maior perceção de intimidade relacional e a existência de satisfação conjugal, protegem a mulher grávida do desenvolvimento de morbilidade psicológica, fornecendo-lhe maior bemestar emocional e maior perceção de suporte aquando da gravidez.

O presente estudo demonstra algumas limitações, as quais podem ter influenciado os resultados obtidos.

Como limitação principal observam-se as características e a dimensão da amostra, dado que esta foi exclusivamente composta por participantes das consultas de preparação para o parto do ACeS Cávado III - Barcelos/Esposende, revelando, portanto, pouca representatividade da população grávida total. Deste modo, conclui-se a existência de um procedimento de amostragem aleatória, o que delimita a generalização dos dados. Note-se que as grávidas que aderem às sessões de preparação para o parto podem-se diferenciar das grávidas em geral, uma vez que, pelos estudos existentes, são em geral mais saudáveis, mais acompanhadas e têm melhor estatuto socioeconómico.

Relativamente aos métodos utilizados na recolha de dados, procurou-se que fossem sempre salvaguardadas as condições mínimas para a participação na in- 
vestigação. No entanto, os questionários de autorrelato encontram-se sujeitos à interpretação de cada participante, sendo que é possível a ocorrência de incompreensões face ao conteúdo dos mesmos e à existência de respostas enviesadas. De outro modo, admite-se ainda a possibilidade de as participantes terem fornecido respostas que se enquadrem no que é socialmente aceite, demonstrando, deste modo, a possibilidade de a desejabilidade social ter enviesado os resultados dos parâmetros avaliados.

Outra limitação do estudo é o desenho transversal, que impossibilita a inferência causal e apenas fornece uma visão do acontecimento em determinado momento.

\section{CONCLUSÕES}

Através dos resultados do presente estudo observaram-se diferenças ao nível da ansiedade entre grávidas multíparas e primíparas, evidenciando as grávidas multíparas maior ansiedade que as primíparas. De facto, observou-se que apesar do conhecimento do processo gravídico e das mudanças que lhe estão associadas, a gravidez representa um período de aumento da ansiedade também para as mulheres multíparas. O maior número de filhos traduz-se num aumento das preocupações e inclusive num agravamento das condições socioeconómicas e da carga efetiva de trabalho.

Deste modo, considera-se que o contexto e as circunstâncias de vida em que a gravidez ocorre assumem maior efeito preditivo que as experiências anteriores de gravidez, potenciando a sintomatologia ansiosa.

Foi ainda observada uma relação positiva entre as preocupações sentidas durante a gravidez e a morbilidade psicológica. Este resultado distingue a importância das preocupações e alerta para a necessidade de proporcionar meios de suporte social adequados que auxiliem a mulher a gerir de modo mais eficaz as preocupações características da gravidez. Porém, demonstra-se ainda necessário incentivar o envolvimento do suporte social (e.g., pai da criança, companheiro, familiares, amigos) a um nível transversal dos cuidados primários, no sentido de reduzir as preocupações associadas ao suporte instrumental e prático aquando da gravidez.

No domínio teórico considera-se pertinente o desenvolvimento de investigações que avaliem o efeito das variáveis relativas ao suporte social, enquanto moderadoras das preocupações sentidas durante a gravidez e dos seus efeitos na morbilidade psicológica da grávida.

Adicionalmente verificou-se que as variáveis da perceção de intimidade relacional e da satisfação conjugal se associam negativamente à morbilidade psicológica da mulher grávida. Estes dados sublinham a importância das variáveis relacionais. De facto, a gravidez constitui para a vida do casal um marco desenvolvimental exigente, no qual os casais experienciam uma nova etapa da vida conjugal. Ao nível teórico, considera-se pertinente a realização de investigações que avaliem o efeito destas variáveis também no companheiro, de modo a estimar as diferenças, ao nível destas variáveis, entre os elementos do casal.

Em suma, recomenda-se que no futuro sejam desenvolvidas, e implementadas, intervenções ao nível do casal e, sobretudo, em mulheres consideradas em risco, avaliando-se ainda a sua eficácia. Ao nível da investigação recomenda-se a realização de estudos de desenho longitudinal com maior incidência no casal e variáveis associadas ao companheiro da grávida.

\section{REFERÊNCIAS BIBLIOGRÁFICAS}

1. Lawrence E, Nylen K, Cobb RJ. Prenatal expectations and marital satisfaction over the transition to parenthood. J Fam Psychol. 2007;21(2) :155-64.

2. World Health Organization. Maternal mental health and child health and development in low and middle income countries: report of the meeting held in Geneva, Switzerland, 30 January - 1 February 2008. Geneva:WHO; 2008. ISBN 9789241597142

3. World Health Organization. Maternal mental health [homepage]. Geneva:WHO; 2014 [cited 2016 Apr 29]. Available from: www.who.int/ mental_health/maternal-child/maternal_mental_health/en/

4. Holton S, Fisher J, Rowe H. Motherhood: is it good for women's mental health? J Reprod Infant Psychol. 2010;28(3):223-39.

5. Bennett HA, Einarson A, Taddio A, Koren G, Einarson TR. Prevalence of depression during pregnancy: systematic review. Obstet Gynecol. 2004;103(4):698-709.

6. Elsenbruch S, Benson S, Rücke M, Rose M, Dudenhausen J, PincusKnackstedt MK, et al. Social support during pregnancy: effects on maternal depressive symptoms, smoking and pregnancy outcome. Hum Reprod. 2007;22(3):869-77.

7. Faisal-Cury A, Araya R, Zugaib M, Menezes PR. Common mental disorders during pregnancy and adverse obstetric outcomes. J Psychosom Obstet Gynaecol. 2010;31(4):229-35.

8. Figueiredo B, Field T, Diego M, Hernandez Reif M, Deeds O, Ascencio A. Partner relationships during the transition to parenthood. J Reprod Infant Psychol. 2008;26(2):99-107. 
9. Gavin NI, Gaynes BN, Lohr KN, Meltzer-Brody S, Gartlehner G, Swinson T. Perinatal depression: a systematic review of prevalence and incidence. Obstet Gynecol. 2005;106(5 Pt 1):1071-83.

10. Røsand GM, Slinning K, Eberhard-Gran M, Røysamb E, Tambs K. Partner relationship satisfaction and maternal emotional distress in early pregnancy. BMC Public Health. 2011;11:161.

11. Ross LE, McLean LM. Anxiety disorders during pregnancy and the postpartum period: a systematic review. J Clin Psychiatry. 2006;67(8):128598.

12. Westdahl C, Milan S, Magriples U, Kershaw TS, Rising SS, Ickovics JR. Social support and social conflict as predictors of prenatal depression. Obstet Gynecol. 2007;110(1):134-40.

13. Alio AP, Lewis CA, Scarborough K, Harris K, Fiscella K.A community perspective on the role of fathers during pregnancy: a qualitative study. BMC Pregnancy Childbirth. 2013;13:60.

14. Campos B, Schetter CD, Abdou CM, Hobel CJ, Glynn LM, Sandman CA. Familialism, social support, and stress: positive implications for pregnant Latinas. Cultur Divers Ethnic Minor Psychol. 2008;14(2):155-62.

15. Rini C, Schetter CD, Hobel CJ, Glynn LM, Sandman CA. Effective social support: antecedents and consequences of partner support during pregnancy. Pers Relatsh. 2006;13(2):207-29.

16. Stapleton LR, Schetter CD, Westling E, Rini C, Glynn LM, Hobel CJ, et al. Perceived partner support in pregnancy predicts lower maternal and infant distress. J Fam Psychol. 2012;26(3):453-63.

17. Lawrence E, Cobb RJ, Rothman AD, Rothman MT, Bradbury TN. Marital satisfaction across the transition to parenthood. J Fam Psychol. 2008;22 (1):41-50.

18. Mitnick DM, Heyman RE, Smith Slep AM. Changes in relationship satisfaction across the transition to parenthood: a meta-analysis. J Fam Psychol. 2009;23(6):848-52.

19. Behringer J, Reiner I, Spangler G. Maternal representations of past and current attachment relationships, and emotional experience across the transition to motherhood: a longitudinal study. J Fam Psychol. 2011;25(2):210-9.

20. Bouchard $\mathrm{G}$. The quality of the parenting alliance during the transition to parenthood. Can J Behav Sci. 2014;46(1):20-8.

21. Conde A, Figueiredo B. Preocupações de mães e pais, na gravidez, parto e pós-parto. Análise Psicológica. 2007;25(3):381-98.

22. Gourounti K, Lykeridou K, Taskou C, Kafetsios K, Sandall J. A survey of worries of pregnant women: reliability and validity of the Greek version of the Cambridge Worry Scale. Midwifery. 2012;28(6):746-53.

23. Green JM, Kafetsios K, Statham HE, Snowdon CM. Factor structure, validity and reliability of the Cambridge Worry Scale in a pregnant population. J Health Psychol. 2003;8(6):753-64.

24. Loke AY, Poon CF. The health concerns and behaviours of primigravida: comparing advanced age pregnant women with their younger counterparts. J Clin Nurs. 2011;20(7-8):1141-50.

25. Carmona Monge FJ, Peñacoba-Puente C, Marín Morales D, Carretero Abellán I. Factor structure, validity and reliability of the Spanish version of the Cambridge Worry Scale. Midwifery. 2012;28(1):112-9.

26. Petersen JJ, Paulitsch MA, Guethlin C, Gensichen J, Jahn A. A survey on worries of pregnant women: testing the German version of the Cambridge worry scale. BMC Public Health. 2009;9:490.

27. Zasloff E, Schytt E, Waldenström U. First time mothers' pregnancy and birth experiences varying by age. Acta Obstet Gynecol Scand. 2007;86(11):1328-36.

28. Redshaw M, Heikkilä K. Ethnic differences in women's worries about labour and birth. Ethn Health. 2011;16(3):213-23.

29. Peñacoba-Puente C, Monge FJ, Morales DM. Pregnancy worries: a longitudinal study of Spanish women. Acta Obstet Gynecol Scand. 2011;90 (9):1030-5.

30. Gourounti K, Anagnostopoulos F, Lykeridou K. Coping strategies as psychological risk factor for antenatal anxiety, worries, and depression among Greek women. Arch Womens Ment Health. 2013;16(5):353-61.

31. Peñacoba-Puente CP, Carmona Monge FJ, Abellán IC, Morales DM. Effects of personality on psychiatric and somatic symptoms in pregnant women: the role of pregnancy worries. Psychol Women Q. 2011;35(2): 293-302.

32. Brockington IF, Macdonald E, Wainscott G. Anxiety, obsessions and morbid preoccupations in pregnancy and the puerperium. Arch Womens Ment Health. 2006;9(5):253-63.

33. Lachance-Grzela M, Bouchard G. Marital status, pregnancy planning, and role overload: a mediated-moderation model of parenting satisfaction. J Fam Psychol. 2009;23(5):739-48.

34. Whisman MA, Davila J, Goodman SH. Relationship adjustment, depression, and anxiety during pregnancy and the postpartum period. J Fam Psychol. 2011;25(3):375-83.

35. Dibaba Y, Fantahun M, Hindin MJ. The association of unwanted pregnancy and social support with depressive symptoms in pregnancy: evidence from rural Southwestern Ethiopia. BMC Pregnancy Childbirth. 2013;13:135.

36. Fisher J, Tran T, La BT, Kriitmaa K, Rosenthal D, Tran T. Common perinatal mental disorders in northern Viet Nam: community prevalence and health care use. Bull World Health Organ. 2010;88(10):737-45.

37. Nasreen HE, Kabir ZN, Forsell Y, Edhborg M. Prevalence and associated factors of depressive and anxiety symptoms during pregnancy: a population based study in rural Bangladesh. BMCWomens Health. 2011;11:22.

38. Boyce P, Condon J, Barton J, Corkindale C. First-Time Fathers' Study: psychological distress in expectant fathers during pregnancy. Aust N Z J Psychiatry. 2007;41(9):718-25.

39. Figueiredo B, Conde A. Anxiety and depression symptoms in women and men from early pregnancy to 3-months postpartum: parity differences and effects. J Affect Disord. 2011;132(1-2):146-57.

40. Ibanez G, Charles MA, Forhan A, Magnin G, Thiebaugeorges O, Kaminski $M$, et al. Depression and anxiety in women during pregnancy and neonatal outcome: data from the EDEN mother-child cohort. Early Hum Dev. 2012;88(8):643-9.

41. Redshaw M, Henderson J. Fathers' engagement in pregnancy and childbirth: evidence from a national survey. BMC Pregnancy Childbirth. 2013;13:70.

42. Rowlands IJ, Redshaw M. Mode of birth and women's psychological and physical wellbeing in the postnatal period. BMC Pregnancy Childbirth. 2012;12:138.

43. Glazier RH, Elgar FJ, Goel V, Holzapfel S. Stress, social support, and emotional distress in a community sample of pregnant women. J Psychosom Obstet Gynaecol. 2004;25(3-4):247-55.

44. Schaefer MT, Olson DH. Assessing intimacy: The Pair Inventory. J Marital Fam Ther. 1981;7(1):47-60. 
45. Moreira H,Amaral A, Canavarro AM. Adaptação do Personal Assessment of Intimacy in Relationships Scale (PAIR) para a população Portuguesa: estudo das suas características psicométricas [Adaptation of the Personal Assessment of Intimacy in Relationships Scale (PAIR) for the Portuguese population: study of its psychometric characteristics]. Psychologica. 2009;(50):339-59. Portuguese

46. Narciso I, Costa ME. Amores satisfeitos, mas não perfeitos. Cad Consult Psicológica. 1996;12:115-130.

47. Nazaré B, Fonseca A, Canavarro AM. Avaliação das preocupações sentidas durante a gravidez: estudos psicométricos da versão portuguesa da Cambridge Worry Scale (CWS). Anal Psicol. 2012;10(1):81-95.

48. Lovibond PF, Lovibond SH. The structure of negative emotional states: comparison of the Depression Anxiety Stress Scales (DASS) with the Beck Depression and Anxiety Inventories. Behav Res Ther. 1995;33(3): 335-43.

49. Pais-Ribeiro JL, Honrado A, Leal IP. Contribuição para o estudo da adaptação portuguesa das Escalas de Ansiedade, Depressão e Stress (EADS) de 21 itens de Lovibond e Lovibond [Contribution to the adaptation study of the Portuguese adaptation of the Lovibond and Lovibond Depression Anxiety Stress Scales (EADS) with 21 items]. Psicol Saúde Doenças. 2004;5(2):229-39. Portuguese

50. Sockol LE, Battle CL. Maternal attitudes, depression, and anxiety in pregnant and postpartum multiparous women. Arch Womens Ment Health. 2015;18(4):585-93.

51. Teixeira C, Figueiredo B, Conde A, Pacheco A, Costa R. Anxiety and depression during pregnancy in women and men. J Affect Disord. 2009;119 (1-3):142-8.

52. Breitkopf CR, Primeau LA, Levine RE, Olson GL, Wu ZH, Berenson AB. Anxiety symptoms during pregnancy and postpartum. J Psychosom Obstet Gynaecol. 2006;27(3):157-62.

53. Bunevicius A, Kusminskas L, Pop VJ, Pedersen CA, Bunevicius R. Screening for antenatal depression with the Edinburgh Depression Scale. J Psychosom Obstet Gynaecol. 2009;30(4):238-43.

54. Dietz PM, Williams SB, Callaghan WM, Bachman DJ, Whitlock EP, Hornbrook MC. Clinically identified maternal depression before, during, and after pregnancies ending in live births. Am J Psychiatry. 2007;164(10): 1515-20.
55. Figueiredo B, Pacheco A, Costa R. Depression during pregnancy and the postpartum period in adolescent and adult Portuguese mothers. Arch Womens Ment Health. 2007;10(3):103-9.

56. Lee AM, Lam SK, Sze Mun Lau SM, Chong CS, Chui HW, Fong DY. Prevalence, course, and risk factors for antenatal anxiety and depression. Obstet Gynecol. 2007;110(5):1102-12.

57. Rallis S, Skouteris H, McCabe M, Milgrom J. A prospective examination of depression, anxiety and stress throughout pregnancy. Women Birth. 2014;27(4):e36-42.

58. Roussis P, Wells A. Psychological factors predicting stress symptoms: metacognition, thought control, and varieties of worry. Anxiety Stress Coping. 2008;21(3):213-25.

59. Mohammad KI, Gamble J, Creedy DK. Prevalence and factors associated with the development of antenatal and postnatal depression among Jordanian women. Midwifery. 2011;27(6):e238-45.

60. Malary M, Shahhosseini Z, Pourasghar M, Hamzehgardeshi Z. Couples communication skills and anxiety of pregnancy: a narrative review. Mater Sociomed. 2015;27(4):286-90.

\section{CONFLITOS DE INTERESSE}

Os autores declaram não possuir qualquer tipo de conflito de interesses.

\section{FINANCIAMENTO}

O trabalho relatado neste manuscrito não foi objeto de qualquer tipo de financiamento externo.

\section{COMISSÃO DE ÉTICA}

O estudo foi realizado após parecer favorável da Comissão de Ética para a Saúde da ARS Norte.

\section{ENDEREÇO PARA CORRESPONDÊNCIA}

\section{Eva Castanheira}

E-mail: evacastanheirapsi@gmail.com

Recebido em 18-05-2016

Aceite para publicação em 18-10-2017 


\section{ABSTRACT \\ RELATIONSHIP BETWEEN PSYCHOLOGICAL MORBIDITY, SOCIODEMOGRAPHIC AND CLINICAL VARIABLES, LACK OF RELATIONAL INTIMACY, MARITAL SATISFACTION AND CONCERNS EXPERIENCED DURING \\ PREGNANCY}

Objectives: to assess the relationship between sociodemographic and clinical variables, relationship intimacy, marital satisfaction, worries during pregnancy and psychological morbidity (anxiety, depression and stress).

Type of study: observational, cross-sectional.

Location: ACES in the northern zone of the country (Barcelos/Esposende- ACES of Cávado III).

Population: women in the second and third trimester of pregnancy.

Methods: the sample was collected during childbirth preparation consultations in the ACES of Cávado III and women completed the questionnaires after the childbirth preparation class.

Results: Greater worries were associated with more psychological morbidity, less perception of relationship intimacy and of marital satisfaction were associated with more psychological morbidity. The perception of relationship intimacy was positively associated with marital satisfaction and negatively associated with worries during pregnancy.

Conclusions: pregnancy can hold considerable risks to the mental health of the woman.

Keywords: Pregnancy; Anxiety; Depression; Stress. 